\title{
The modeling of the process of grain material outflow from a hopper bin with a lateral outlet
}

\author{
Artyom Doroshenko ${ }^{1, *}$, Andrey Butovchenko ${ }^{2}$, and Lyubov Gorgadze ${ }^{1}$ \\ ${ }^{1}$ Don state technical university, Agroindustrial Department, 344000 Rostov-on-Don, Russian \\ Federation \\ ${ }^{2}$ Don state technical university, Social and human Department, 344000 Rostov-on-Don, Russian \\ Federation
}

\begin{abstract}
Cleaning and sorting of agricultural materials are the essential processes in agricultural production. Many researchers have carried out important work on the study of optimizing the parameters of grain cleaning machines. One of these parameters, affecting the quality of machine, is the grain material outflow characterized by patterns of input velocities into the separation zone. The article describes the advantages and disadvantages of methods for determining the rate of grain material outflow expiration. Here is shown the model of heterogeneous bulky environment of outflow from the hopper with a lateral outlet in a software complex EDEM Simulation. The analysis of the obtained results with the use of contact model HertzMindlin (no slip) with RVD Rolling Friction has demonstrated a significant sparseness of grain material outflow velocities in a steady state of $0,61 \mathrm{~m} / \mathrm{s}$ to $0,72 \mathrm{~m} / \mathrm{s}$ with an average $0,687 \mathrm{~m} / \mathrm{s}$ for the specified parameters of a grain cleaning machine. The described method can be used to solve the problem of determining the rate of expiration of grain material in the design of grain cleaning machines with the subsequent optimization of their operation.
\end{abstract}

\section{Introduction}

Currently, the timing of new agricultural machinery release can be greatly reduced by using instrumentation that enables at the design stage to carry out simulation of the work of its individual component parts. When designing pneumatic separating channels for the separation of grain heap representing heterogeneous bulk medium in calculations the distribution irregularity of input velocities across the width of the hopper pneumatic separating channel (at different points they are different) is not taken into account neither the shape of the particles themselves or the position of the center of gravity of the particle is considered, etc. These inaccuracies in the calculation do not allow designing an optimum construction of a pneumatic separating machine which meets the specified requirements. Also, designing without the use of visualization software simulation does not allow to look inside the process and to optimize the design and operation of the device. In this case it is necessary to make a laboratory stand or a prototype and improve the design. These actions

\footnotetext{
*Corresponding author: 79094190528@yandex.ru
} 
lead to an increase in the cost of the project and significantly extend the time of new machinery launching into the market.

Cleaning and sorting of agricultural materials is the essential process in agricultural production. Many researchers conducted important researches in this field to improve the efficiency and quality of grain material pneumatic separation, as well as to reduce losses caused by further cleaning. During the process of grain material pneumatic separation in a vertical pneumatic conveyor a lot of private technological operations are performed. Here, we consider the process of entering grain material into a pneumatic channel working zone. One of the parameters directly affecting the quality of separation of grain material into fractions is the velocity of its input into a pneumatic channel working zone. This parameter has been studied and described by many scientists.

The opening of pneumatic separator hopper flap results in a decrease in vertical pressure which can be accompanied by an increase in lateral pressure depending on the height-towidth ratio $(\mathrm{H} / \mathrm{B})$. This excess pressure occurred immediately after the start of the expiration, after which the pressure and velocity remained almost constant until a significant decrease in the level of grains [1,2,3, and 4].

\section{Vertical Pneumatic Separator}

\subsection{Vertical Pneumatic Separator Construction}

At preliminary processing of grain material after harvesting it is expedient to allocate the biologically defective grain and weed impurities by an air stream. Thus, fractionation of grain heap by means of pneumatic separating systems enables to select the seeds of grain material with higher germinating capacity. In the design of the pneumatic separator, the input of material cleaned is made passively, by filling the hopper and opening the flap. Passive devices include various pitched boards, grids, and sieves. These devices are simple in design, do not require additional power consumption, and have little impact on the damage of grain material supplied to the pneumatic separator.

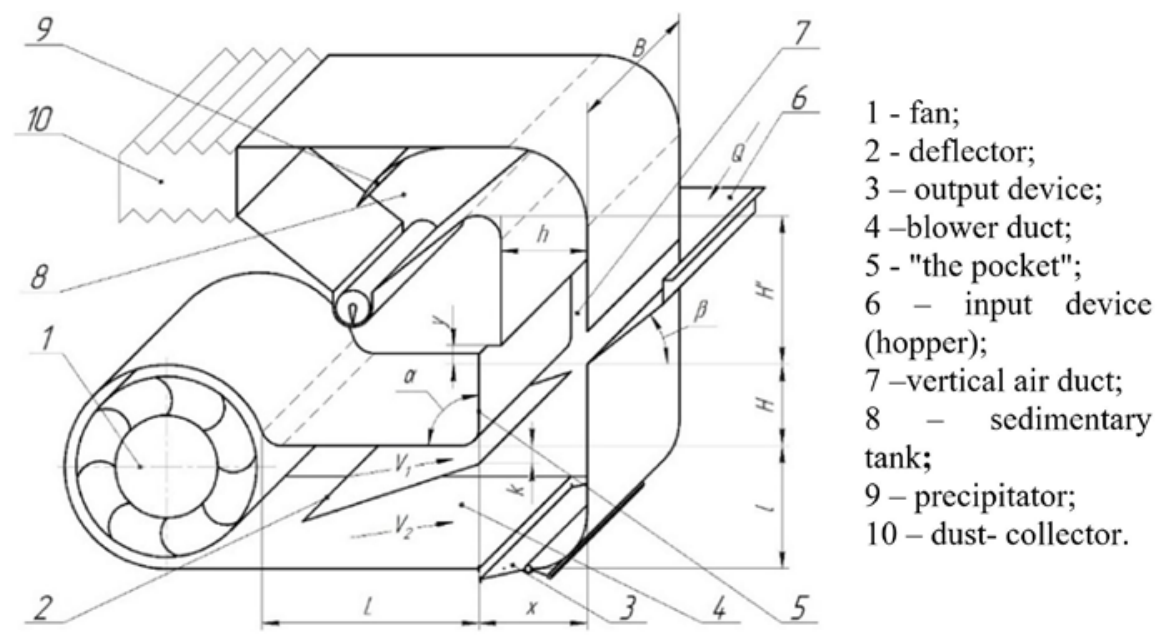

Fig. 1. The general view of the pneumatic separator. 


\subsection{Analysis of the Method of Solving the Problem}

The rate of grain material outflow can be determined experimentally. One of the methods is to determine time of unloading a given amount of grain. This method has low accuracy in the overlay error mechanisms determine the time measurements, grain weighing and inaccuracies of the mechanism of overlapping outlet of the hopper pneumatic separator.

Another method to determine the rate grain material outflow is high-speed videorecording of the grain material outflow process [5]. This method enables to determine the outflow velocity indirectly and at the edges of the hopper by calculating the path traversed by the grain per time unit.

The determination of particle flow velocity is possible with the use of X-ray tomography scanning [6]. Due to the low-spatial resolution of electrical capacity tomography or scanning speed limitation of standard X-ray CT systems, it is extremely challenging to measure the flow velocity and possible centrifugal effects of granular materials flow effectively.

The solution to this problem can be the use of joint DEM CFD calculations (DEM Discrete Element Modeling - family of numerical methods for calculating a large number of particles. CFD - computational fluid dynamics is a subsection of continuum mechanics which includes a set of physical, mathematical and numerical methods designated to calculate outflow processes characteristics [7]. To obtain the most realistic calculation, software systems enable to simulate heterogeneous bulk medium having properties as close as possible to the original. EDEM Simulation was chosen as a software package for further calculations.

\section{DEM Simulations}

\subsection{Particle Contact Model}

The alternative particle contact models in EDEM environment are the Hertz-Mindlin (no slip), Hertz-Mindlin (no slip) with RVD Rolling Friction, Hertz-Mindlin with JKR, and Hertz-Mindlin with bonding, linear cohesion, and linear spring contact models. Considering computational efficiency and the successful experience in related literature [7], the most popular Hertz-Mindlin (no slip) with RVD Rolling Friction contact model was selected in the present study. This contact model type was developed based on the work of Mindlin [10]. The methods for determining the variables used in the software package are presented in a variety of papers $[8,9$, and 10$]$ and in order to avoid the information duplication are not presented in this paper.

\subsection{Particle Model}

In this article the particle of grain material of wheat is taken as the object of research. The three-dimensional model of wheat grain shown as an ellipsoid with the length of $6,3 \mathrm{~mm}$ and the diameter of $3,1 \mathrm{~mm}$ is illustrated in figure 2. The particle generator was used to create of wheat grain heap with different grain lengths ranging from 5,6 $\mathrm{mm}$ to $8,6 \mathrm{~mm}$. The distribution of grain wheat corresponds to the normal law of distribution (Fig. 2). The particle contact parameters and the mechanical properties of the materials used in the EDEM simulation are respectively listed in Tables 1 and 2. 

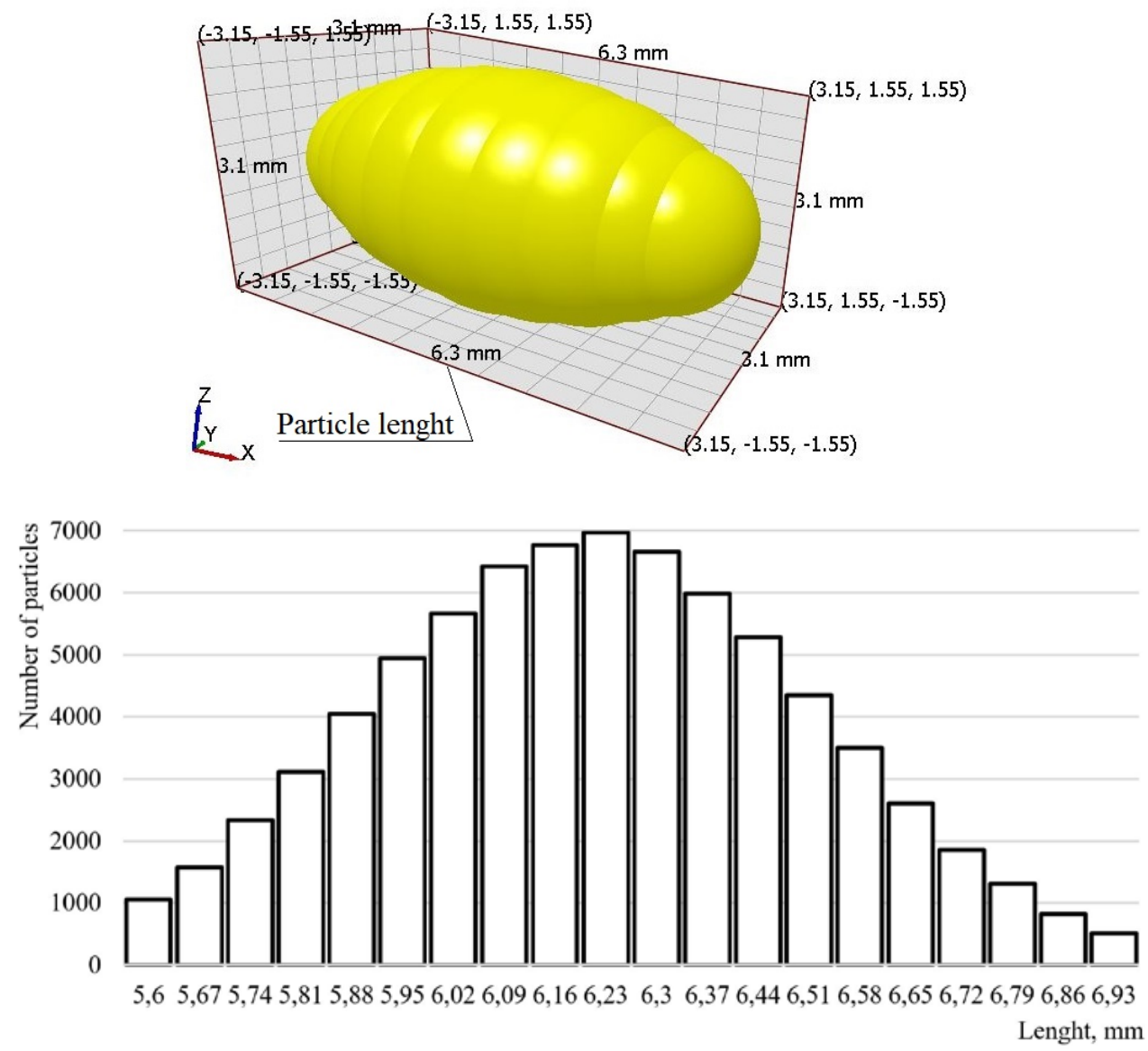

Fig. 2. Three-dimensional model of wheat grain (a) and the length distribution of wheat grains in the grain material.

Table 1. Mechanical properties in the materials used in the EDEM simulation.

\begin{tabular}{|c|c|c|}
\hline Material properties & Wheat grain & Steel \\
\hline Poisson's ratio & 0,35 & 0,3 \\
\hline Shear modulus $(\mathrm{MPa})$ & 100 & 700 \\
\hline Density $\left(\mathrm{kg} / \mathrm{m}^{3}\right)$ & 1350 & 7800 \\
\hline
\end{tabular}

Table 2. Particle contact parameters used in the EDEM simulation.

\begin{tabular}{|c|c|c|}
\hline Contact parameters & Wheat grain $\backslash$ wheat grain & Wheat grain $\backslash$ steel \\
\hline Coefficient of restitution & 0,2 & 0,5 \\
\hline Coefficient of static friction & 0,545 & 0,5 \\
\hline Coefficient of rolling friction & 0,1 & 0,05 \\
\hline
\end{tabular}

\subsection{EDEM Simulation Condition}

Figure 3 illustrates the component parts used in simulation, referred to as the bottom of the hopper, the hopper walls, and the height of the flap opening of the hopper. At the beginning of calculation, wheat grains were generated, and after filling the hopper, a flap was opened. The width of the hopper is $100 \mathrm{~mm}$. To study the characteristics of the outflow of grain the 
boundary conditions in the area of material output were removed from the hopper by $100 \mathrm{~mm}$ in the direction of axis $\mathrm{x}$ and $\mathrm{z}$.

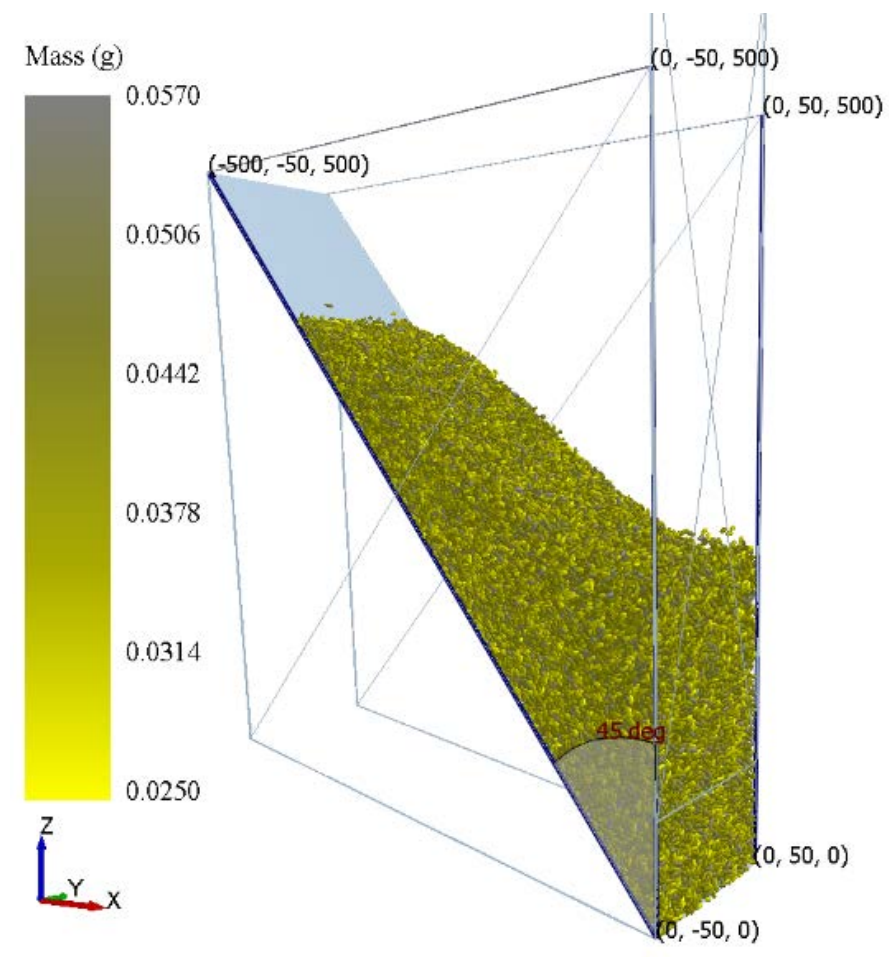

Fig. 3. Simulation condition in EDEM.

The angle of inclination of the hopper bottom is equal to 45 degrees, and the height of the flap opening is $80 \mathrm{~mm}$. These parameters correspond to the mode of pneumatic separator operation with a capacity of 8 tons per hour, the scheme of which was presented earlier.

\section{Result and Discussion}

When modelling grain material was generated and set to a static position in the interval from 0 to 1 second. The flap opened with the speed of $2 \mathrm{~m} / \mathrm{s}$ and the grain material began to flow under gravity. We have chosen the sample numerical values of velocity vectors of the grain material expiration at a time step of $0.01 \mathrm{sec}$. The consolidated graph of grain material velocity at the time of expiration is presented in figure 4 .

The average outflow velocity in the steady-state process of expiration was $6,87 \mathrm{~m} / \mathrm{s}$, and the range of varying velocities of $0,61 \mathrm{~m} / \mathrm{s}$ to $0,72 \mathrm{~m} / \mathrm{s}$. The sparseness of values was from $0,248 \mathrm{~m} / \mathrm{s}$ to $0,84 \mathrm{~m} / \mathrm{s}$ which is conditioned by grain material low velocities in the areas of grain material contact with the bottom of the hopper at the point of entry and increased velocities of grain material in the upper layers of the particle flow.

The experimental bulk velocity of the grain material at the same parameters was 0,376 $\mathrm{m} / \mathrm{s}$. In order to avoid the obtained data duplication the experimental values are presented in the article [11]. Such significant differences in velocities are conditioned by that grain material that is being in contact with the walls of the hopper slows down and the rest of the grain under the influence of gravity moves freely. 


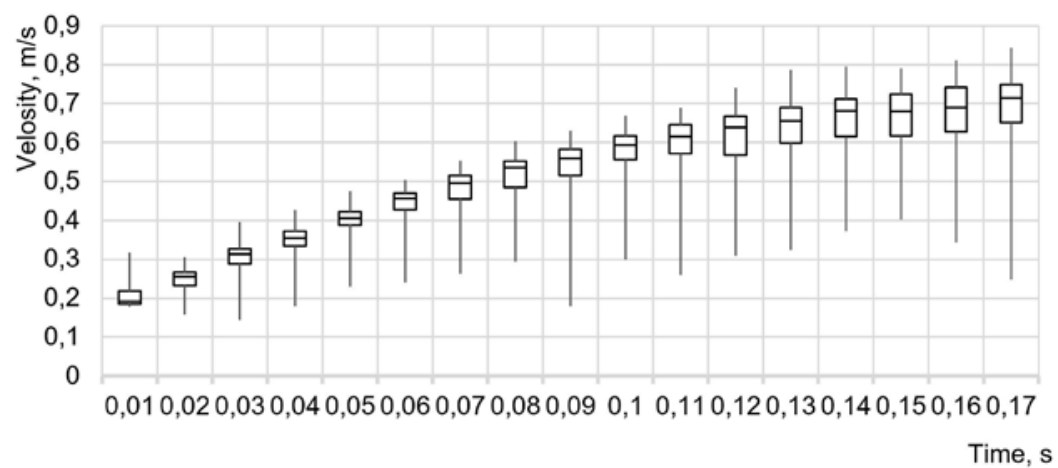

Fig. 4. Graph of the dependence of grain material velocity at the time of expiration.

\section{Conclusion}

In this article we have considered the process of grain material outflow from the hopper with a lateral outlet. The model of expiry was developed with the use of the software EDEM Simulation. The following conclusions were obtained for modelling variable-length particles with the specified parameters of the hopper:

- The sparseness of averaged velocities of the expiration of the grain material is large and significantly differs from theoretical and experimental values;

- The method described can be used to solve the problem of determining the rate grain material outflow in the design of grain cleaning machines with the subsequent optimization of their operation;

- The obtained array of values can serve as initial data for modelling of particle and air flow interaction.

\section{References}

1. A. Couto, A. Ruiz, P.J. Aguado, Comput. Electron. Agric., 85, pp. 174-187 (2012)

2. H.B. Manbeck, M.G. Goyal, G.L. Nelson, M.G. Singh, Dynamic overpressures in model bins during emptying (Pap. ASAE for Winter Meet., USA; 13-16 December 1977)

3. Q. Zhang, M.G. Britton, R. Jaremek, J. Agric. Eng. Res., 56(2), pp. 111-119 (1993)

4. M. Molenda, J. Horabik, I.J. Ross, Trans. ASAE, 38(6), pp. 1869-1873 (1995)

5. T. Börzsönyi, Z. Kovács, Phys. Rev. E, 83(3), 032301 (2011)

6. S. Waktola, A. Bieberle, F. Barthel, M. Bieberle, U. Hampel, K. Grudzień, L. Babout, Exp. Fluids, 59, 69 (2018)

7. H. Li, Y. Li, Z. Tang, Trans. CSAM, 83(3), 032301 (2012)

8. Z. Ma, Y. Li, L. Xu, Comput. Electron. Agric., 118, pp. $92-93$ (2015)

9. J.M. Boac, M.E. Casada, R.G. Maghirang, J.P. Harner, Trans. ASABE, 53(4), pp. 1201-1216 (2010)

10. P. Cundall, O. Strack, Geotech., 29(1), pp. 47-65 (1979)

11. J.I. Ermolyev, A.V. Butovchenko, A.A. Doroshenko, Bulletin DSTU, 1, 122-134 (2014) 